\title{
Analgesia preventiva con microdosis de ketamina en pacientes operados con anestesia general
}

\author{
Preemtive analgesia with micro doses of ketamine \\ in patients under general anesthesia
}

Yanexis Martínez Sánchez1, Guillermo Capote Guerrero², Yurisnel Ortiz Sánchez, Marta Rosa Rosabal Sadin ${ }^{4}$, Kenia Dalia León Paz ${ }^{5}$

\begin{abstract}
Introduction: Acute postoperative pain is a complex physiological reaction to tissue injury or disease. Ketamine, an NMDA receptor antagonist, is the only intravenous anesthetic with hypnotic, analgesic and amnesic properties. Objective: To evaluate the effectiveness of the administration of ketamine microdosis in patients operated with general anesthesia. Methods: A prospective, quasi-experimental, controlled, double-blind study was conducted in patients operated under general anesthesia by the General Surgery service at the University Carlos Carlos de Céspedes University Hospital in Bayamo, in the period from January 2016 to December 2018. Results: Age was homogeneous in both groups. The female sex prevailed in both groups without significant differences. There is a similarity in the mean ENV scores in the study group throughout the entire follow-up, never reaching 2 points. On the contrary, the patients in the control group, obtained throughout the follow-up an average score around 3 points. A lower opioid demand is observed in the group to which the ketamine microdose was administered. Hemodynamic parameters (heart rate, systolic and diastolic blood pressure) remained stable throughout the study period without statistically significant differences between the two. Conclusions: The administration of ketamine microdosis, as preventive analgesia in patients operated with general anesthesia, has proven effective in the control of acute postoperative pain.
\end{abstract}

\footnotetext{
Especialista de primer grado en Medicina General Integral y Anestesiología y Reanimación.

Especialista de $2^{\text {do }}$ grado en Anestesiología y Reanimación. Diplomado en terapia intensiva. Profesor auxiliar.

Dr. C. Lic. en farmacología. Profesor titular.

Especialista de $2^{\text {do }}$ grado en Anestesiología y Reanimación. Máster en urgencias médicas. Profesor asistente.

Especialista de primer grado en Medicina General Integral y Anestesiología y Reanimación. Profesor asistente.
}

Fecha de recepción: 10 de octubre de 2019

Fecha de aceptación: 05 de enero de 2020

ORCID

http://orcid.org/0000-0003-2841-8348

Correspondencia:

Guillermo Capote Guerrero

gcapote@infomed.sld 


\section{RESUMEN}

Introducción: El dolor postoperatorio agudo es una reacción fisiológica compleja a la lesión tisular o enfermedad. La ketamina, antagonista del receptor NMDA, es el único anestésico intravenoso con propiedades hipnóticas, analgésicas y amnésicas. Objetivo: Evaluar la efectividad de la administración de microdosis de ketamina en pacientes operados con anestesia general. Métodos: Se realizó un estudio prospectivo, cuasi experimental, controlado, doble ciego en pacientes operados con anestesia general por el Servicio de Cirugía General en el Hospital Provincial Universitario "Carlos Manuel de Céspedes" de Bayamo, en el período comprendido desde enero de 2016 hasta diciembre de 2018. Resultados: La edad fue homogénea en ambos grupos. El sexo femenino predominó en ambos grupos sin diferencias significativas. Hay una similitud en las puntuaciones medias de la ENV en el grupo estudio a lo largo de todo el seguimiento, nunca llegando a los 2 puntos. Por el contrario, los pacientes del grupo control, obtuvieron a lo largo de todo el seguimiento una puntuación media en torno a los 3 puntos. Se observa una demanda de opioide inferior en el grupo al que se le administró la microdosis de ketamina. Los parámetros hemodinámicos (frecuencia cardíaca, presión arterial sistólica y diastólica) permanecieron estables durante todo el periodo de estudio sin diferencias estadísticamente significativas entre ambos. Conclusiones: La administración de microdosis de ketamina, como analgesia preventiva en pacientes operados con anestesia general, ha demostrado ser efectiva en el control del dolor agudo postoperatorio.

\section{Palabras clave:}

Ketamina, analgesia preventiva, dolor postoperatorio

\section{Introducción}

E I dolor postoperatorio agudo es una reacción fisiológica compleja a la lesión tisular o enfermedad. Se trata de una manifestación de las reacciones autonómicas, psicológicas y conductuales que resultan de una experiencia molesta, sensorial y emocional indeseada[1].

Su manejo es fundamental para mejorar la calidad de atención y disminuir las complicaciones postoperatorias que aumenten la morbilidad, mortalidad y tiempo de hospitalización[2].

La incisión quirúrgica, es un desencadenante de respuestas de inflamatorias y simpáticas, esto conlleva a un aumento en la liberación de catecolaminas, consumo de oxígeno y aumento de la actividad neuroendocrina, la cual puede conducir a complicaciones cardiovasculares, respiratorias, metabólicas, gastrointestinales, inmunológicas y psicológicas[3].

La principal recomendación para el tratamiento del dolor postoperatorio es la utilización de técnicas de analgesia multimodal que puede incluir la administración de dos o más medicamentos por la misma o distinta vía con diferentes mecanismos de acción para el control del dolor[4]-[7].
En cuanto a analgesia endovenosa se refiere, es amplio el arsenal de medicamentos disponibles para manejo de dolor agudo postoperatorio según la intensidad del estímulo quirúrgico al que fue expuesto un paciente determinado[8].

La ketamina, es el único anestésico intravenoso con propiedades hipnóticas, analgésicas y amnésicas. Tiene un período de latencia corto, brinda un nivel anestésico estable cuando se emplea tanto en perfusión continua como en bolos, potencializando el efecto analgésico opioide sin requerir ser empleada a las dosis hipnóticas que se emplearon en algún tiempo. De esta forma, al emplearse la ketamina a dosis bajas, se ha llegado a obtener los beneficios completos de la analgesia profunda, posterior a su administración en bolos únicos a dosis subanestésicas[8]-[10].

Hay evidencia de que la ketamina ocupa los receptores opioides en el cerebro y en la médula espinal, lo que podría explicar algunos de sus efectos analgésicos. El efecto analgésico a nivel espinal parece deberse a una inhibición de la actividad neuronal de la asta dorsal[9],[10],[11],,[12].

El fundamento para el uso de la ketamina en el control del dolor agudo y crónico, se inicia desde hace 
varios años, cuando era utilizado como complemento de la anestesia regional para procedimientos quirúrgicos, bloqueo de nervios, anestesia epidural o espinal y control del dolor en curaciones de pacientes con quemaduras. Este fármaco neuromodulador, a bajas dosis, se ha utilizado con efectividad para el control del dolor postoperatorio refractario a opioides, de igual manera puede ser administrado por vía oral, intranasal o transdérmica, con un elevado perfil de seguridad y escasos efectos adversos[13],[14].

Se parte de la hipótesis que: la administración de microdosis de ketamina como adyuvante de la anestesia general es una manera efectiva, segura, sencilla, para lograr analgesia preventiva y disminuir el consumo de analgésicos opiodes durante el trans y postoperatorio.

El objetivo de la realización de esta investigación consiste en evaluar la efectividad de la administración de microdosis de ketamina en pacientes operados con anestesia general.

\section{Método}

Se realizó un estudio prospectivo, cuasi experimental, controlado, doble ciego en pacientes operados con anestesia general por el Servicio de Cirugía General en el Hospital Provincial Universitario "Carlos Manuel de Céspedes" de Bayamo, en el período comprendido desde enero de 2016 hasta diciembre de 2018.

\section{Universo y muestra}

El universo de estudio estuvo formado por 4.039 pacientes intervenidos quirúrgicamente en el salón de cirugía general en el período de estudio. Para seleccionar el tamaño de la muestra, se tuvo en cuenta el error tipo I de $5 \%(\alpha=5 \%)$ con una desviación estándar que no excedió de $25 \%$, con el empleo del paquete estadístico Epi Info versión 6.1, arrojando un tamaño de la muestra de 86 pacientes.

\section{Criterios de selección de la muestra}

La selección de pacientes se realizó en la consulta de anestesia, una vez anunciada su intervención quirúrgica, por medio de interrogatorio y exploración física, revisión de exámenes complementarios con la finalidad de detectar función de órganos y sistemas y detectar cualquier anormalidad en ellos. Así mismo se les dio una breve explicación sobre el concepto de la escala análoga visual.

\section{Criterios de inclusión}

- Pacientes de cualquier sexo, entre 20 a 60 años, con estado físico ASA I y II, intervenidos quirúrgicamente de manera electiva y a los cuales se les administró anestesia general, y brindaron su consentimiento a participar en la investigación.

\section{Criterios de exclusión}

- Pacientes con incapacidad para comprender el uso de la escala visual análoga (EVA).

- Se excluyeron a aquellos con tratamiento previo, hipersensibilidad o alergia a AINEs o a la ketamina, con enfermedad renal o hepática, cardiovascular y/o pulmonar severa, con antecedentes de terapia anticoagulante o discrasias sanguíneas, de úlcera gastroduodenal o contraindicaciones para uso de fármacos antiinflamatorios.

\section{Tratamiento}

Se crearon dos grupos de pacientes, un grupo estudio: Grupo ketamina (K) y un grupo control: Grupo placebo $(\mathrm{P})$. Los pacientes en cada grupo desconocían qué fármaco es el que se le administró con el objetivo de evitar que se condicionaran a tener efectos positivos y así recolectar datos reales.

En ambos grupos se procedió de la siguiente manera: Previa oxigenación con $\mathrm{fiO}_{2}$ al $100 \%$ durante 3 minutos por máscara facial, se realizó la inducción anestésica con: Propofol $2 \mathrm{mg} / \mathrm{kg}$, fentanilo $3 \mathrm{mcg} / \mathrm{kg}$. Se logró la relajación muscular para la laringoscopia con atracurio 0,5 mg/Kg.

De 5 a 10 minutos antes de la incisión quirúrgica se administró al grupo estudio ketamina 0,20 mg/kg por vía EV y al grupo control se le administró 5 cc de solución salina al 0,9\%.

El mantenimiento anestésico en ambos grupos se realizó con propofol en infusión continua.

Se utilizó como relajante muscular no despolarizante el atracurio a $0,25 \mathrm{mg} / \mathrm{kg}$ cada 25 o 30 minutos según necesidades.

El fentanilo se administró a razón de 1 a $2 \mathrm{mcg} / \mathrm{kg}$ cada 30 minutos según requerimientos hemodinámicos.

De 30 a 40 minutos antes de terminar la operación se les administró a los pacientes de ambos grupos por vía EV diclofenaco sódico a $1 \mathrm{mg} / \mathrm{kg}$.

En la unidad de cuidados posoperatorios $30 \mathrm{mi}-$ nutos después de la llegada del paciente a la misma y una vez recobrada completamente la conciencia, se evaluó en ambos grupos la intensidad del dolor y los 
requerimientos de analgesia de rescate con la Escala Visual Análoga. Esta se evaluó a los 30 minutos, 2 horas, 6 horas, 12 horas y 24 horas después de la operación. Por lo que requirió de la visita del anestesiólogo a la sala en la cual se encuentre hospitalizado el paciente. Además, se evaluó durante estas visitas la aparición de alguna complicación.

Las dosis de rescate fueron pautadas de la siguiente manera:

Dolor leve: cuando la escala análoga visual (EVA) oscila entre 0 y 3, se administró diclofenaco a 1 mg/ kg por vía IM o EV.

Dolor moderado: cuando la EVA se valoró entre 4 y 7 . En este caso se administró tramadol $1 \mathrm{mg} / \mathrm{kg}$ endovenoso.

Dolor intenso: cuando la EVA se valoró entre 7 y 10. Se administró petidina a $1 \mathrm{mg} / \mathrm{kg}$ endovenoso.

\section{Criterios de evaluación de la efectividad:}

1- Respuesta satisfactoria

- Cuando al realizar la valoración en el posoperatorio el paciente no refirió dolor o presente dolor ligero.

- Cuando no apareció complicaciones.

2- Respuesta no satisfactoria

- Cuando al realizar la valoración en el posoperatorio el paciente refirió dolor moderado o severo.

- Cuando apareció alguna complicación o efecto adverso.

\section{Análisis estadístico}

Se hizo análisis de frecuencia de variables de interés que permitieron establecer datos de correlación categórica y numérica sin agrupar entre el uso de analgesia preventiva y la intensidad de dolor o no presencia de este, se utilizó pruebas de asociación de significancia estadística en la variable categórica Chi cuadrado, $V$ de Cramer y el intervalo de contingencia y en el caso de los datos sin agrupar se hizo análisis con el uso de t de Student para dos muestras independientes, con un margen de error a esperar de $5,0 \%$ es decir, $\alpha=0,05$

\section{Resultados}

En este estudio fueron incluidos 86 pacientes, divididos en dos grupos de 43 pacientes. Ambos grupos fueron comparables: la edad fue homogénea en ambos grupos, con una media aritmética de 39,8 años para el grupo experimental ( $D E \pm 13,2$ años), y de 40,1 años para el grupo control ( $D E \pm 13,5$ años). El sexo femenino predominó en ambos grupos sin diferencias significativas: $81,4 \%$ para el grupo experimental y $86,1 \%$ para el control. Así mismo no hubo diferencia significativa en la valoración ASA, tiempo de anestesia, tiempo de cirugía, tipo de programación y EVA preoperatorio.

Los resultados globales obtenidos, así como los correspondientes a los diferentes momentos del estudio (30 minutos, 2 horas, 8 horas, 24 horas y 48 horas) se muestran en la Tabla 1, en relación a la calidad analgésica, evaluada por la Escala Numérica Verbal (ENV).

Para realizar un análisis pormenorizado de los mismos, inicialmente se muestran los resultados globales, expresados como media de las puntuaciones obtenidas en cada uno de los momentos del estudio en los dos grupos objeto de estudio (Tabla 2). En todos ellos las cifras de ENV han sido menores en el grupo al que se le administró la microdosis de ketamina, mostrando uniformemente diferencias estadísticamente significativas.

En dicha tabla, se observa que prácticamente hay una similitud en las puntuaciones medias de la ENV en el grupo estudio a lo largo de todo el seguimiento, nunca llegando a los 2 puntos, siendo incluso esta puntuación menor en el postoperatorio inmediato, obteniendo menos de 1 punto de media. Por el contrario, los pacientes del grupo control, obtuvieron a lo largo de todo el seguimiento una puntuación media en torno a los 3 puntos.

Al valorar la efectividad de las técnicas analgésicas cuantificando los requerimientos de opioides que precisaron los pacientes durante las primeras 48 horas del postoperatorio. En la Tabla 3 se muestra el número de bolos de opioides demandados por el paciente estratificados según el tiempo de seguimiento y grupo de tratamiento, observándose una demanda muy inferior en el grupo al que se le administró la microdosis de ketamina alcanzando diferencias estadísticamente significativas entre ambos grupos en todos los momentos del estudio, con una $p$-valor inferior a 0,0001.

Asimismo, en esta tabla se puede observar que en el grupo al que se le administró las microdosis de ketamina, a lo largo de todo el seguimiento, la demanda de bolos de opioide, fue casi homogénea; no así en el grupo control donde, a partir de las 8 horas, esta demanda aumenta de forma considerable.

Los parámetros hemodinámicos (frecuencia cardíaca, presión arterial sistólica y diastólica) permanecieron estables durante todo el periodo de estudio sin diferencias estadísticamente significativas entre ambos grupos y con respecto a los valores hemodiná- 


\begin{tabular}{|c|c|c|c|c|c|c|}
\hline \multirow[t]{2}{*}{ Tiempo } & \multirow[t]{2}{*}{ ENV } & \multicolumn{2}{|c|}{ Experimental } & \multicolumn{2}{|c|}{ Control } & \multirow[t]{2}{*}{$p$} \\
\hline & & No. & $\%$ & No. & $\%$ & \\
\hline \multirow[t]{4}{*}{30 minutos } & 0 & 30 & 69,8 & 13 & 30,2 & 0,0000 \\
\hline & $1-3$ & 12 & 27,9 & 16 & 37,2 & \\
\hline & $4-6$ & 1 & 2,3 & 10 & 23,3 & \\
\hline & $7-10$ & 0 & 0,0 & 4 & 9,3 & \\
\hline \multirow[t]{4}{*}{2 horas } & 0 & 22 & 51,2 & 10 & 23,3 & 0,0000 \\
\hline & $1-3$ & 18 & 41,9 & 16 & 37,2 & \\
\hline & $4-6$ & 3 & 7,0 & 15 & 34,9 & \\
\hline & $7-10$ & 0 & 0,0 & 2 & 4,7 & \\
\hline \multirow[t]{4}{*}{8 horas } & 0 & 15 & 34,9 & 5 & 11,6 & 0,0000 \\
\hline & $1-3$ & 25 & 58,1 & 29 & 67,4 & \\
\hline & $4-6$ & 3 & 7,0 & 9 & 20,9 & \\
\hline & $7-10$ & 0 & 0,0 & 0 & 0,0 & \\
\hline \multirow[t]{4}{*}{24 horas } & 0 & 7 & 16,3 & 2 & 4,7 & 0,0000 \\
\hline & $1-3$ & 30 & 69,8 & 28 & 65,1 & \\
\hline & $4-6$ & 6 & 14,0 & 13 & 30,2 & \\
\hline & $7-10$ & 0 & 0,0 & 0 & 0,0 & \\
\hline \multirow[t]{4}{*}{48 horas } & 0 & 21 & 48,8 & 2 & 4,7 & 0,0000 \\
\hline & $1-3$ & 18 & 41,9 & 34 & 79,1 & \\
\hline & $4-6$ & 4 & 9,3 & 7 & 16,3 & \\
\hline & $7-10$ & 0 & 0,0 & 0 & 0,0 & \\
\hline
\end{tabular}

\begin{tabular}{lccc}
\hline \multicolumn{4}{c}{ Tabla 2. Calidad analgésica. Valores medios de ENV } \\
\hline & Experimental & Control & p-valor \\
30 minutos & $0,57(1,1)$ & $2,73(2,5)$ & 0,00 \\
2 horas & $1,42(1,4)$ & $3,14(1,8)$ & 0,00 \\
8 horas & $1,86(1,4)$ & $2,71(1,3)$ & 0,00 \\
24 horas & $1,89(1,3)$ & $2,84(1,4)$ & 0,00 \\
48 horas & $1,91(1,4)$ & $2,86(1,5)$ & 0,00 \\
Total & $1,43(1,0)$ & $2,84(1,3)$ & 0,00 \\
\hline
\end{tabular}

Fuente: Historia clínica y planilla de vaciamiento de datos.

micos basales. Ambos grupos mostraron frecuencias respiratorias similares a las basales sin que se apreciaran diferencias significativas entre grupos, igualmente, las saturaciones de oxígeno por pulsioximetría se mantuvieron en un rango de normalidad clínica, entre el $95 \%-98 \%$ durante las $24 \mathrm{~h}$ de estudio. El nivel de sedación fue similar en ambos grupos de estudio, no se evidenció ningún caso de depresión respiratoria postoperatoria.

En cuanto a la incidencia de efectos adversos no hubo diferencias significativas entre ambos grupos, la incidencia de náuseas y vómitos postoperatorios fueron: grupo experimental 6 (13,95\%), grupo control 9 (20,93\%). Un paciente del grupo experimental presentó diaforesis. Ningún paciente presentó alucinaciones o disforia. 


\begin{tabular}{|c|c|c|c|c|}
\hline & Experimental & Control & Total & p-valor \\
\hline 30 minutos & $0,04(0,2)$ & $1,02(1,4)$ & $0,69(1,0)$ & 0,00 \\
\hline 2 horas & $0,39(0,5)$ & $1,29(1,1)$ & $0,87(1,0)$ & 0,00 \\
\hline 8 horas & $0,50(0,6)$ & $2,14(2,1)$ & $1,39(1,8)$ & 0,00 \\
\hline 24 horas & $0,86(1,0)$ & $4,71(7,4)$ & $2,99(5,7)$ & 0,00 \\
\hline 48 horas & $0,77(1,2)$ & $4,34(7,6)$ & $2,71(5,9)$ & 0,00 \\
\hline Total & $2,50(2,3)$ & $13,62(16,1)$ & $8,54(13,1)$ & 0,00 \\
\hline
\end{tabular}

Los resultados se muestran en media (DS); Fuente: Historia clínica y planilla de vaciamiento de datos.

Tabla 4. Distribución de pacientes de ambos grupos de tratamiento según la evolución clínica

\begin{tabular}{|c|c|c|c|c|c|c|}
\hline \multirow{3}{*}{$\begin{array}{l}\text { Evolución } \\
\text { satisfactoria }\end{array}$} & \multirow{2}{*}{\multicolumn{2}{|c|}{$\begin{array}{c}\text { Grupos } \\
\text { Experimental }\end{array}$}} & \multicolumn{2}{|c|}{ Control } & \multicolumn{2}{|c|}{ Total } \\
\hline & & & & & & \\
\hline & No. & $\%$ & No. & $\%$ & No. & $\%$ \\
\hline Sí & 36 & 83,72 & 24 & 55,81 & 60 & 69,77 \\
\hline No & 7 & 16,28 & 19 & 44,19 & 26 & 30,23 \\
\hline Total & 43 & 100,00 & 43 & 100,00 & 86 & 100,00 \\
\hline
\end{tabular}

$\chi^{2}=6,671 ; g l=1 ; p=0,0000$ (muy significativo); Fuente: Historia clínica y planilla de vaciamiento de datos.

La hipótesis planteada al inicio de la investigación se comprobó, evidenciando la efectividad de la administración de microdosis de ketamina, como adyuvante de la anestesia general, es una manera efectiva, segura, sencilla, para lograr analgesia preventiva y disminuir el consumo de analgésicos opiodes durante el trans y postoperatorio; siendo mayor el número de pacientes con respuesta satisfactoria en el grupo estudio 36 pacientes $(83,72 \%)$, que en el control 24 pacientes $(55,81 \%)$ mostraron respuesta satisfactoria. Resultados significativos desde el punto de vista estadístico también se arrojó al aplicar la prueba Chi cuadrado $\left(\chi^{2}\right)$ (Tabla 4$)$.

\section{Discusión}

La analgesia preventiva busca mantener un control de las variables responsables de la sensibilización central cuando no es posible utilizar otras técnicas de analgesia[15],[16],[17].

El uso de la Ketamina previo, es una opción asociada a otras técnicas continuas de analgesia por vía intravenosa como son los opioides[14],[18]. La disminución evidente del dolor en los pacientes donde fue utilizada la ketamina corrobora una vez más los re- sultados de varios investigadores que utilizaron analgesia preventiva con ketamina encontrando similares resultados a los nuestros[19],[20],[21]-[24].

Elia y Tramer[25] realiza una revisión sistemática cuantitativa cuyo objetivo fue estudiar el papel de la ketamina en el dolor postoperatorio, el ahorro de opioides y la incidencia de efectos adversos. Se incluyeron 53 estudios, de los cuales 16 utilizaban la vía IV , demostrando que ésta a una dosis mediana de 0,4 $\mathrm{mg} / \mathrm{kg}$ en 850 adultos, disminuyó la intensidad del dolor por escala análoga visual.

En un estudio publicado en 2014 por McNicol y colaboradores[26] que realiza un metaanálisis para encontrar la efectividad de la ketamina en la disminución de la prevalencia y severidad del dolor postquirúrgico persistente, aplicada por cualquier vía en el período perioperatorio. Los estudios presentaron alta heterogeneidad, y la vía de administración intravenosa demostró de forma estadísticamente significativa reducción en el desarrollo de dolor postquirúrgico persistente a los 3 y 6 meses.

En el estudio la disminución en el consumo de opiodes pudiera ser, al menos en parte, como resultado de sus efectos sobre el comportamiento que no se refleja en la intensidad del dolor. Sin embargo, su uso a bajas dosis $<2,5 \mathrm{mcg} / \mathrm{kg} / \mathrm{min}$ en pacientes postope- 
rados ha sido reportado como seguro, sin alucinaciones o fallas en el funcionamiento cognitivo[11],[13].

En una investigación, realizado por Sánchez y colaboradores[27], similar al nuestro en donde se incluyeron 38 pacientes, distribuidos en tres grupos, para demostrar la eficacia de la ketamina, para disminuir los requerimientos analgésicos en el transoperatoria y posoperatorio, sin causar efectos adversos importantes. El grupo A recibió ketamina a 0,25 mg, el grupo $B$ ketamina a $0,5 \mathrm{mg}$ y el grupo $C$ un placebo (solución fisiológica). Encontraron que la administración preincisional de ketamina en dosis subanestésicas, es segura y efectiva para proveer analgesia intraoperatoria y postoperatoria inmediata en pacientes pediátricos sometidos a cirugías de adenotonsilectomías, además, ninguna de las dosis causó efectos colaterales importantes.
Finalmente, se evaluó la presencia de efectos adversos posterior a la administración de los fármacos estudiados, obteniéndose que en la mayoría de los casos no se presentaron, solo en los grupos de ketamina se evidenció en un número significativo de pacientes, prolongación del efecto sedante, manifestado por somnolencia. Sánchez y colaboradores[27], no reportaron efectos adversos indeseables en sus estudios. Mientras que Bell y colaboradores[28], refirieron que estos fueron leves o no se registraron.

Se concluye que la administración de microdosis de ketamina, como analgesia preventiva en pacientes operados con anestesia general, ha demostrado ser efectiva en el control del dolor agudo postoperatorio durante las primeras 48 horas, lográndose una disminución en el consumo de opioides, tanto de modo global como en cada uno de los momentos del estudio.

\section{Referencias}

1. Kraychete Durval Campos, Sakata Rioko Kimiko, Lannes Leticia de Oliveira Carvalho, Bandeira Igor Dórea, Sadatsune Eduardo Jun. Postoperative persistent chronic pain: what do we know about prevention, risk factors, and treatment. Rev. Bras. Anestesiol. [Internet]. 2016 Oct [cited 2019 Jan 03] ; 66( 5 ): 505-512. Available from: http://www. scielo.br/scielo.php?script=sci_ arttext\&pid=S0034$70942016000500505 \& \operatorname{lng}=$ en. https://doi.org/10.1016/j.bjane.2014.12.005

2. Humble SR, Dalton AJ, Li L. A systematic review of therapeutic interventions to reduce acute and chronic postsurgical pain after amputation, thoracotomy or mastectomy. Eur J Pain. 2015;19:451-465. https://doi. org/10.1002/ejp.567

3. Chinchilla-Hermida Paola Andrea, Baquero-Zamarra David Ricardo, Guerrero-Nope Carlos, Bayter-Mendoza Eduardo Francisco. Incidence of chronic post-surgical pain and its associated factors in patients taken to inguinal hernia repair. Rev. colomb. anestesiol. [Internet]. 2017 Dec [cited 2019 Jan 02] ; 45(4):291-299.Available from: http://www.scielo. org.co/scielo.php?script=sci_ arttext\&pid=S012033472017000400291\&lng=en. https://doi.org/10.1016/j. rcae.2017.07.002

4. Thapa P and Euasobhon P. Chronic postsurgical pain: current evidence for prevention and management. Korean J Pain. 2018 Jul; 31(3): 155-173. https://doi. org/10.3344/kjp.2018.31.3.155

5. Reddi D. Preventing chronic postoperative pain. Anaesthesia 2016; 71: 64-71. https://doi. org/10.1111/anae.13306

6. Clarke $\mathrm{H}$, Poon M, Weinrib A, Katznelson R, Wentlandt K, Katz $J$. Preventive analgesia and novel strategies for the prevention of chronic post-surgical pain. Drugs. 2015;75:339-51. https://doi. org/10.1007/s40265-015-0365-2

7. Richebé $P$, Julien $M$, Brulotte $V$. Potential strategies for preventing chronic postoperative pain: a practical approach: Continuing Professional Development. Canadian Journal of Anesthesia/ Journal canadien d'anesthésie 2015; 62: 1329-41. https://doi. org/10.1007/s12630-015-0499-4

8. Gorlin A, Rosenfeld DM, and

Ramakrishna H. Intravenous subanesthetic ketamine for perioperative analgesia. J Anaesthesiol Clin Pharmacol. 2016 Apr-Jun; 32(2): 160-167. https://doi. org/10.4103/0970-9185.182085

9. Steven P. Cohen, Anuj Bhatia, Asokumar Buvanendran, Eric $S$. Schwenk, Ajay D. Wasan, Robert W. Hurley, Eugene R. Viscusi, Samer Narouze, Fred N. Davis, Elspeth C. Ritchie, Timothy R. Lubenow, William M. Hooten. Consensus Guidelines on the Use of Intravenous Ketamine Infusions for Chronic Pain From the American Society of Regional Anesthesia and Pain Medicine, the American Academy of Pain Medicine, and the American Society of Anesthesiologists. Reg Anesth Pain Med. 2018 Jul 43(5): 521-546. Published online 2018 Jun 7. https://doi.org/10.1097/ AAP.0000000000000808

10. Eric S. Schwenk, Eugene R. Viscusi, Asokumar Buvanendran, Robert W. Hurley, Ajay D. Wasan, Samer Narouze, Anuj Bhatia, Fred N. Davis, William M. Hooten, Steven P. Cohen. Consensus Guidelines on the 
Use of Intravenous Ketamine Infusions for Acute Pain Management From the American Society of Regional Anesthesia and Pain Medicine, the American Academy of Pain Medicine, and the American Society of Anesthesiologists. Reg Anesth Pain Med. 2018 Jul 43(5): 456466. Published online 2018 Jun 7. https://doi.org/10.1097/ AAP.0000000000000806

11. Cogan J, Lalumiere G, VargasSchaffer G, et al. Low-dose intravenous ketamine for postcardiac surgery pain: effect on opioid consumption and the incidence of chronic pain. Ann Card Anaesth 2017;20:395-8. https:// doi.org/10.4103/aca.ACA_54_17

12. Othman $A H$, El-Rahman AM, El Sherif F. Efficacy and safety of ketamine added to local anesthetic in modified pectoral block for management of postoperative pain in patients undergoing modified radical mastectomy. Pain Physician 2016;19:485-94.

13. Bowers KJ, McAllister KB, Ray M, Heitz $C$. Ketamine as an adjunct to opioids for acute pain in the emergency department: a randomized controlled trial. Acad Emerg Med. 2017;24(6):676685. https://doi.org/10.1111/ acem. 13172

14. Brian M. Radvansky, Khushbu Shah, Anant Parikh, Anthony N. Sifonios, Vanny Le, and Jean D. Eloy, "Role of Ketamine in Acute Postoperative Pain Management: A Narrative Review," BioMed Research International, vol. 2015, Article ID 749837, 10 pages, 2015. https://doi. org/10.1155/2015/749837. https://doi org/10.1155/2015/749837

15. Tiippana E, Hamunen K, Heiskanen T, Nieminen T, Kalso E, Kontinen VK. New approach for treatment of prolonged postoperative pain: APS Out-
Patient Clinic. Scandinavian Journal of Pain. 2016;12:1924 https://doi.org/10.1016/j. sjpain.2016.02.008

16. Garduño-López AL, NucheCabrera E, Monroy-Álvarez C. Dolor postoperatorio: optimización del manejo en el contexto perioperatorio. Rev Mex Anest. 2016;39:S16-S19.

17. Chapman CR, Vierck CJ. The transition of acute postoperative pain to chronic pain: an integrative overview of research on mechanisms. J Pain. 2017;18:359. e1-359.e38. https://doi. org/10.1016/j.jpain.2016.11.004

18. Bolívar Trujillo MA. Administración de Ketamina intravenosa en el perioperatorio para el control del dolor agudo postoperatorio en pacientes adultos sometidos a cirugía de resección primaria de neoplasias sólidas. Tesis. Universidad Nacional de Colombia. Bogotá D.C., Colombia, 2017.

19. Quemé Figueroa AS. Eficacia de la ketamina versus S-ketamina para el manejo del dolor agudo en niños quemados. Tesis. Universidad de San Carlos de Guatemala. Enero 2016.

20. Panos Zanos, Ruin Moaddel, Patrick J. Morris, Lace M. Riggs, Jaclyn N. Highland, Polymnia Georgiou, Edna F. R. Pereira, Edson X. Albuquerque, Craig J. Thomas, Carlos A. Zarate, Jr., Todd D. Gould. Ketamine and Ketamine Metabolite Pharmacology: Insights into Therapeutic Mechanisms. Pharmacol Rev. 2018 Jul; 70(3): 621-660. Published online 2018 Jul. https://doi. org/10.1124/pr.117.015198

21. Hidalgo Velásquez José, Ortega Valdés María Elena, Morales Jiménez Lesbel, Pimienta Peguero Milagro. Microdosis de ketamina en la prevención del dolor postoperatorio. Rev Ciencias Médicas [Internet]. 2005 Sep [citado 2018 Di 19] ; 9( 3 ): 11-20. Disponible en: http://scie- lo.sld.cu/scielo.php?script=sci_ arttext\&pid=S156131942005000300003\&lng=es.

22. Peyton PJ, Wu C, Jacobson $T$, et al. The effect of a perioperative ketamine infusion on the incidence of chronic postsurgical pain - a pilot study. Anaesth Intensive Care 2017; 45: 459465. https://doi.g/10.1177/0310 057X1704500408

23. Schug SA and Peyton P. Does perioperative ketamine have a role in the prevention of chronic postsurgical pain: the ROCKet trial. Br J Pain. 2017 Nov; 11(4): 166-168. https://doi. rg/10.1177/2049463717736076

24. Ye F, Wu Y, Zhou C. Effect of intravenous ketamine for postoperative analgesia in patients undergoing laparoscopic cholecystectomy: A meta-analysis. Medicine (Baltimore). 2017;96(51):e9147. https://doi.org/10.1097/ MD.0000000000009147

25. Elia N, Tramèr MR. Ketamine and postoperative pain-a quantitative systematic review of randomised trials. Pain. 2005;113(1-2):6170. https://doi.org/10.1016/j. pain.2004.09.036

26. McNicol ED, Schumann R, Haroutounian S. A systematic review and meta-analysis of ketamine for the prevention of persistent post-surgical pain. Acta Anaesthesiol Scand. 2014;58(10):1199-213. https:// doi.org/10.1111/aas.12377

27. Sánchez S, Viteri M, Valero A. Ketamina en dosis subanestésicas como efecto analgésico en niños sometidos a adenotonsilectomías: estudio preliminar. Rev. Venez. Anestesiol;2003; 8(1):26-32.

28. Bell R, Dahl J, Moore R, Kalso E. Perioperative ketamine for acute postoperative pain. Cochrane Database Syst Rev. 2006;(1): Art. No.: CD004603 (Actualizado 2010). https://doi.org/10.1002/14651858. CD004603.pub2 